\title{
ROLE OF HEALTH CARE PROFESSIONALS IN PREVENTATIVE ACTIVITIES AND VACCINATION PROGRAMS DURING OUTBREAKS
}

\author{
SÁNDOR BALOGH ${ }^{1,2 *}$, ReNÁtA PAPP ${ }^{1,2}$, CSILla BusA ${ }^{1,3}$ and ÁGNES CsiKós ${ }^{1}$ \\ ${ }^{1}$ Institute of Primary Health Care, University of Pécs Medical School, Pécs, Hungary \\ ${ }^{2}$ Family Physicians, European Union of General Practitioners, Rome, Italy \\ ${ }^{3}$ Social Fact Institute, Pécs, Hungary
}

(Received: 1 April 2016; accepted: 25 August 2016)

\begin{abstract}
Purpose: The general purpose of TELL ME study was to give an insight into the experiences of European family physicians with management of H1N1 pandemic flu. Methods: Qualitative research methods (focus group discussions, oneto-one interviews, and online data collection) were used to explore family doctors' opinion and suggestions. Overall 158 family physicians took part in the study from six European countries. Results: Family doctors' most important experience was that the official campaign was not able to compensate negative effects of the mass media. Due to the poor evidence-based information about new vaccines, it was difficult to convince the public and some health care professionals too. Lack of unified directives - under unclear circumstances - made the routine patient care more difficult and hampered the collaboration between different health care providers. Family physicians felt a pressure from health authorities to achieve high immunization rate, but got only a little support from them. Despite the difficulties, vaccination program was a success, mainly among high-risk population. For better handling of a future pandemic, Hungarian family physician made many general and practical suggestions.
\end{abstract}

Keywords: pandemic, H1N1, family physician, vaccination, communication

*Corresponding author; E-mail: balogh.sandor@pte.hu

This is an open-access article distributed under the terms of the Creative Commons Attribution License, which permits unrestricted use, distribution, and reproduction in any medium for non-commercial purposes, provided the original author and source are credited. 


\section{Introduction}

The appearance of new global threats from previously unknown or little known viruses such as SARS, H5N1, H1N1, and Ebola reminds us that viruses have not been conquered but continue to threaten human worldwide [1]. Although health organizations worldwide better prepared for an outbreak than ever before, and the risk of such an outbreak turning into a pandemic is low, the danger of a global pandemic is rising again and again. The WHO declares the Zika virus as a public health emergency, and it shows why we need to improve health communication continuously.

In the European Commission's Assessment Report on EU-wide pandemic vaccine strategies, they state that "pandemic planners need to find a way to get health care professionals more actively engaged so their valuable knowledge and experiences can be considered in the planning process. Without their engagement and support, the effectiveness of vaccine communications is inhibited." [2].

Although family physicians were expected to maintain a central role during the swine flu in 2009 [3, 4], for many of them H1N1 was the first pandemic situation in their professional life [5]. On the other hand, not much is published on health care workers communication requirements including evidence-based information about vaccine safety [6, 7], key messages, and communication with health authorities $[8,9]$.

The aim of the TELL ME study was to identify new challenges and communication requirements of health care professionals (mainly family physicians) and to develop new methods concerning outbreak communication [10].

\section{Methods}

TELL ME was a qualitative study, comprising focus group discussions, oneto-one interviews, and online data collection among family physicians in Europe. Overall 158 family doctors were included from six European countries: 32 from Hungary, 29 from the United Kingdom, 29 from Italy, 25 from Romania, 20 from Belgium, and 23 from Denmark. Countries were selected in order to achieve a geographical spread across the EU and to ensure in-depth data on social and cultural environmental factors from different regions of Europe. Focus group discussions were video or audio recorded and transcribed verbatim. Transcripts were analyzed using thematic analysis methods as follows: (1) general overviewing of interview transcripts in order to identify characteristic patterns of themes; (2) generating codes; (3) searching for themes; and (4) exploration of connections and correlations [11]. This study focuses on the Hungarian results of TELL ME study. (Direct quotes are italicized, and respondents are identified by ID codes.) 


\section{Results}

Management of pandemic $f u$

Hungarian family physicians had mixed overall opinion about general management of pandemic flu. Many aspects of the campaign (including objectives, tools, logistic, financing, responsibility, etc.) were not clear to them from the start.

At the beginning of pandemic influenza, many different directives and recommendations were published by health authorities, health organizations, and professional associations (WHO-national-local, gynaecologists, immunologists, etc.). Lack of unified directives made the routine patient care more difficult and hampered the cooperation between different health care providers. Family physicians had to make responsible decisions day by day under unclear circumstances (e.g., vaccination of pregnant women, institutionalized children, patients with mercury, or egg allergy), and they got only a little support from the authorities.

"Doctors cannot be left alone in outbreaks! They should get very clear tasks and answers who to vaccinate who not, what are the features of the vaccine." (H21)

Physicians were under pressure from patients and health authorities as well. Patients wished to get protection without any risks, meanwhile health authorities wanted to achieve higher and higher immunization rates.

"I suggested vaccine uptake for everyone. National Public Health and Medical Officer Service made a really strong campaign. They called us regularly and demanded progress report on vaccine uptake. We were under pressure. I felt that vaccination should be compulsory." (H7)

\section{Official information vs. mass media}

Official information from the national health authorities was delayed especially in comparison to the mass media. Doctors would have required more information about size of epidemic, ways of prevention, etc., before the public health media campaigns. Vaccination program against H1N1 was a subject to controversial discussions during the pandemic, and physicians also wanted to access scientific evidence, e.g., clinical studies and comparative studies about effectiveness and side effects of the new vaccines.

Problems-related mass media were common experiences of Hungarian family physicians. Main topic of media debate among health experts was vaccine safety, and many worrying reports were published on this topic in tabloid newspapers as well. Due to uncertainty, ignorance, and misinformation, much 
false information was associated with the vaccine (e.g., urban legends about chip in vaccine in order to monitor people or to exterminate retirees). Necessity of vaccination was also raised due to relative mildness of the pandemic flu. After the bird flu which did not affect Hungary, a part of the population was absolutely apathetic.

Factors influencing vaccine uptake in Hungary

Patients had access to vaccines via family physicians, at the vaccination centers, and pharmacies with prescription. In accordance with the recommendations, Hungarian family doctors focused on target groups who received vaccines free of charge. Free vaccine (Fluval) was provided by the National Public Health and Medical Officer Service but many aspects of logistics were unclear, and vaccine delivery caused extra costs for family doctors.

Immunization rate was average or higher than seasonal flu vaccine uptake in most investigated practices. Vaccination of at-risk patients (old people, chronic patients, etc.) was the most successful thanks to their greater willingness and free vaccines.

"Many patients - especially older patients - came and the vaccines were available. People accepted the necessity of vaccination." (H2)

In contrast, people outside the target groups have been difficult to reach. Those patients had to pay for the vaccine; therefore, many patients were not vaccinated. Furthermore, most practices were not able to provide a separate area for patients with flu symptoms, so doctors designated separate office hours in the evenings or on Saturday mornings. Their extra time was appreciated by healthy patients - but was not reimbursed by the National Health Insurance Fund.

Hard-to-reach group of patients were young people and pregnant women in Hungary. People belonging to Roma minority were also found to be a difficult group to persuade. Family physicians also experienced that highly qualified people had lower trust in vaccines, and they demanded correct and evidence-based information about the vaccine and its side effects. Good personal relationship, knowledge of health and family conditions of their patients, and personalized communication helped family physicians to convince their reluctant patients.

"The personal conviction and persuasion is the key. The connection should be so good that patients accept the doctor's suggestion. But it is a heavy work for many years." ( $\mathrm{H} 2$ )

Most Hungarian family doctors also took up the vaccine and had their partners, children, and vulnerable family members vaccinated, and persuaded patients with a sense of civic duty. 
Family physicians' recommendations

For better handling of a future pandemic, Hungarian family physician made many essential suggestions. An effective media campaign has to be implemented on a professional basis, using evidence-based information provided by health authorities, and health professionals. Involvement of professionals increases credibility, and need for well-trained professionals who are experienced public speakers.

To avoid panic, misleading information has to be corrected by the "official" media straightway. In view of changing media usage, patterns must be considered as power and effects of social media and have to use it in a professional way.

Many suggestions were also given by doctors relating to information flow and cooperation, mainly improving collaboration between different health care professionals from the start. They emphasized the importance of involving family physicians in a more effective way (e.g., set up an anti-crisis group).

Improving access to shot is a key factor: separate, reimbursed office hours and weekend clinics should be provided for healthy patients. Most health care workers suggested enlarging the target group who get vaccine for free and cheaper vaccine (max. 1,000 Ft/3 EUR) for not at-risk patients.

In order to improve vaccine uptake in "hard-to-reach" groups, specific approaches are needed, such as tailored, face-to-face doctor-patient communication, and specific information leaflets for different patient groups. Involving patients' organizations was also mentioned as an effective way to reach at-risk patients.

\section{Discussion}

During infection outbreaks, adequate communication is crucial in order to reduce the spread of disease and to avoid panic. According to Hungarian family physicians, national and local health authorities should have handled better the situation during the 2009/2010 pandemic flu. Although doctors needed official and mainly evidence-based information, authorities informed them only after a big media campaign. Patients were scared and health care workers had no persuasive information and professional tools to calm the "hysteria." Misleading media communication, self-proclaimed experts, rumors, and conspiracy theories [12], focusing on sensationalism over science, have caused many negative effects. "Rumors typically emerge in a context of asymmetries of power. As access to knowledge and definitions of what is true or not rest upon limited groups of experts, those left aside develop alternative ways to express their opinion. Rumors provide parallel information, especially when official communication is limited to a top-down approach." [13]. Family physicians' general perception was that the 
negative media effects undermined the credibility of the official communication, and it was the key factor of poor vaccine uptake.

Hungarian family physicians reported problems-related coordination between professionals, too. Health care workers had different knowledge and opinions about risk and side effects of the vaccine, and despite the official recommendations, many doctors were against vaccination (e.g., gynaecologists' doubts about vaccination of pregnant women). Not only evidence-based information is needed, but it is also "important to empower health care workers through supporting the skills of acquiring and using evidence-based information" [3].

A simpler vaccination plan, which is clearly communicated and consistent with regard to both providers and the public, would be required. Shortly, after the influenza A (H1N1) 2009 pandemic began, the U.S. government provided guidance to state and local authorities to assist decision-making, recommending local, collaborative planning, and posing a series of questions regarding epidemiology, the impact on the health care system, and locally determined feasibility and acceptability of non-pharmaceutical strategies - unfortunately such efforts were clearly absent for the EU region [14].

Communication failures-related influenza H1N1 pandemic showed that we need to learn to exploit the potential that the info society may offer in terms of evidence-based and participatory communication. National pandemic plans around the world have been guided by government, public health agencies, and other experts, but countries lately worldwide are encouraged to use a societal approach to pandemic planning as well. Research increasingly shows the importance of public engagement in pandemic planning. The community-based participatory approach can aid in understanding community perspectives and values, helps accept recommended actions by the public and execute at the community level $[15,16]$.

Results of the TELL ME project also confirmed that family physicians play a major role in preventative activities during pandemic: they serve as the patient's first entry point into the health care system and also serve as important opinion leaders [3]. Family doctors have a high credibility in patients' eyes, higher than the public trust in the governmental communication. The 2009 influenza H1N1 pandemic raised a disturbing issue that is the limited compliance of health care professionals with vaccination plans. In Europe, the proportion of health care professionals accepting immunization is consistently under $50 \%$ [17]. The picture is almost the same everywhere, e.g., Germany [18], United Kingdom [19], Spain [20], Italy [21], and Turkey [22], confirming findings [23-26] showing that health care professionals tend to have negative attitude toward flu vaccination. Still more telling is another study [27] that shows: health care professionals, as parents, are likely to under vaccinate against H1N1 virus their children. It is difficult to convince the public to get vaccinated when the people administering the vaccines 
mistrust the vaccine and are not getting vaccinated themselves or their children. The concern about vaccine safety and distrust of health authorities are the commonest reasons given for low compliance with vaccination by health care workers. Better communication strategies to improve vaccination acceptance by the general population and by the health care workers are urgently required $[3,7]$.

\section{Acknowledgement}

TELL ME Collaborative Project has received funding from the European Union's FP7 programme under grant agreement no. 278723.

\section{Conflict of Interest}

The authors have no potential conflicts to declare.

\section{References}

1. Strauss, J. H., Strauss, E. G.: Viruses and Human Disease, 2nd Edition. Academic Press, New York, 2007, p. 1.

2. European Commission: Assessment report on EU-wide pandemic vaccine strategies. CRISMART Swedish National Defence College, Stockholm, 115 pp. (2010).

3. Hidiroglu, S., Ay, P., Topuzoglu, A., Kalafat, C., Karavus, M.: Resistance to vaccination: The attitudes and practices of primary healthcare workers confronting the H1N1 pandemic. Vaccine 28, 8120-8124 (2010).

4. Eizenberg, P.: The general practice experience of the swine flu epidemic in Victoria Lessons from the front line. Med J Aust 191, 151-153 (2009).

5. Baker, M.: Communicating in a crisis: The H1N1 influenza pandemic. Br J Gen Pract 60 , 237-238 (2010).

6. Hashim, A., Jean-Gilles, L., Hegermann-Lindencrone, M., Shaw, I., Brown, C., NguyenVan-Tam, J.: Did pandemic preparedness aid the response to pandemic (H1N1) 2009? A qualitative analysis in seven countries within the WHO European Region. J Infect Public Health 5, 286-296 (2012).

7. Blasi, F., Aliberti, S., Mantero, M., Centanni, S.: Compliance with anti-H1N1 vaccine among healthcare workers and general population. Clin Microbiol Infect 18, 37-41 (2012).

8. Whelan, J., Greenland, K., Rondy, M., van der Hoek, W., Robert-Du, Ry., van Beest Holle, M.: Case registry systems for pandemic influenza A(H1N1)pdm09 in Europe: Are there lessons for the future? Euro Surveill 17, 15-23 (2012).

9. Van Kerkhove, M. D., Ferguson, N. M.: Epidemic and intervention modelling - A scientific rationale for policy decisions? Lessons from the 2009 influenza pandemic. Bull World Health Organ 90, 306-310 (2012).

10. Transparent Communication in Epidemics: Learning Lessons from experience, delivering effective Messages, providing Evidence (TELL ME) Project (GA No 278723). Available at http://www.tellmeproject.eu/. 
11. Green, J., Thorogood, N.: Qualitative Methods for Health Research. Sage Publications Ltd., London, 2004, pp. 173-200.

12. Cirhinlioğlu, F. G., Cirhinlioğlu, Z.: Social representations of H1N1 influenza A (Swine Flu). Rev Sociol 40, 273-295 (2010).

13. Barrelet, C., Bourrier, M., Burton-Jeangros, C., Schindler, M.: Unresolved issues in risk communication research: The case of the H1N1 pandemic (2009-2011). Influenza Other Respir Viruses 7, 114-119 (2013).

14. Barrios, L. C., Koonin, L. M., Kohl, K. S., Cetron, M.: Selecting nonpharmaceutical strategies to minimize influenza spread: The 2009 Influenza A (H1N1) pandemic and beyond. Public Health Rep 127, 565-571 (2012).

15. Charania, N. A, Leonard, J. S., Tsuji, L. J. S.: A community-based participatory approach and engagement process creates culturally appropriate and community informed pandemic plans after the $2009 \mathrm{H} 1 \mathrm{~N} 1$ influenza pandemic: Remote and isolated First Nations communities of sub-arctic Ontario, Canada. BMC Public Health 12, 268 (2012).

16. Cyril, S., Smith, B. J, Possamai-Inesedy, A., Renzaho, A. M. N.: Exploring the role of community engagement in improving the health of disadvantaged populations: A systematic review. Glob Health Action 8, 29842 (2015).

17. VENICE Survey: Vaccine European New Integrated Collaboration Effort (VENICE) Project. Available at http://venice.cineca.org/.

18. Leitmeyer, K., Buchholz, U., Kramer, M., Schenkel, K., Stahlhut, H., Köllstadt, M., Haas, W., Meyer, C.: Influenza vaccination in German health care workers: Effects and findings after two rounds of a nationwide awareness campaign. Vaccine 24, 7003-7008 (2006).

19. Blank, P. R., Freiburghaus, A. U., Schwenkglenks, M., Szucs, T. D.: Trends in influenza vaccination coverage rates in the United Kingdom over six seasons from 2001-2 to 2006-7. Euro Surveill 13, 19074 (2008).

20. Vírseda, S., Restrepo, M. A., Arranz, E., Magán-Tapia, P., Fernández-Ruiz, M., de la Cámara, A. G., Aguado, J. M., López-Medrano, F.: Seasonal and Pandemic A (H1N1) 2009 influenza vaccination coverage and attitudes among health-care workers in a Spanish University Hospital. Vaccine 28, 4751-4757 (2010).

21. La Torre, G., Di Thiene, D., Cadeddu, C., Ricciardi, W., Boccia, A.: Behaviours regarding preventive measures against pandemic H1N1 influenza among Italian healthcare workers, October 2009. Euro Surveill 14, 7-9 (2009).

22. Rachiotis, G., Mouchtouri, V. A., Kremastinou, J., Gourgoulianis, K., Hadjichristodoulou, C.: Low acceptance of vaccination against the 2009 pandemic influenza A(H1N1) among healthcare workers in Greece. Euro Surveill 15, 8-14 (2010).

23. Weingarten, S., Riedinger, M., Bolton, L. B., Miles, P., Ault, M.: Barriers to influenza vaccination acceptance: A survey of physicians and nurses. Am J Infect Control 17, 202 207 (1989).

24. Hak, E.: Negative attitude of highly educated parents and health care workers towards future vaccinations in the Dutch childhood vaccination program. Vaccine 23, 3103-3107 (2005).

25. National Foundation for Infectious Disease: Improving Influenza vaccination rates in health care workers, 2004. Available at www.nfid.org/pdf/publications/hcwmonograph.pdf.

26. Watanakunakorn, C., Ellis, G., Gemmel, D.: Attitude of healthcare personnel regarding influenza immunization. Infect Control Hosp Epidemiol 14, 17-20 (1993).

27. Torun, S. D., Torun, F., Catak, B.: Healthcare workers as parents: Attitudes toward vaccinating their children against pandemic influenza A/H1N1. BMC Public Health 10, 596 (2010). 